\title{
Investigating Axonal Damage in Multiple Sclerosis by Diffusion Tensor Spectroscopy
}

\author{
Emily T. Wood, ${ }^{1,2}$ Itamar Ronen, ${ }^{3}$ Aranee Techawiboonwong, ${ }^{4}$ Craig K. Jones, ${ }^{5,6}$ Peter B. Barker, ${ }^{5,6}$ Peter Calabresi, \\ Daniel Harrison, ${ }^{7}$ and Daniel S. Reich ${ }^{1,6,7,8}$ \\ ${ }^{1}$ NeuroImmunology Branch (NINDS), National Institutes of Health, Bethesda, Maryland 20892, ${ }^{2}$ Department of Neuroscience, Johns Hopkins University \\ School of Medicine, Baltimore, Maryland 21205, ${ }^{3}$ C.J. Gorter Center for High Field MRI, Department of Radiology, Leiden University Medical Center, 2333 \\ ZA Leiden, Netherlands, ${ }^{4}$ Department of Electrical Engineering, Mahidol University, Salaya, Nakhon Pathom 73170, Thailand, ${ }^{5}$ F.M. Kirby Research Center \\ for Functional Brain Imaging, Kennedy Krieger Institute, Baltimore, Maryland 21205, and ${ }^{\circ}$ Department of Radiology and ${ }^{7}$ Department of Neurology, Johns \\ Hopkins University School of Medicine, Baltimore, Maryland 21287, and ${ }^{8}$ Department of Biostatistics, Johns Hopkins University School of Public Health, \\ Baltimore, Maryland 21205
}

Sensitive and specific in vivo measures of axonal damage, an important determinant of clinical status in multiple sclerosis (MS), might greatly benefit prognostication and therapy assessment. Diffusion tensor spectroscopy (DTS) combines features of diffusion tensor imaging and magnetic resonance spectroscopy, allowing measurement of the diffusion properties of intracellular, cell-type-specific metabolites. As such, it may be sensitive to disruption of tissue microstructure within neurons. In this cross-sectional pilot study, diffusion of the neuronal metabolite $\mathrm{N}$-acetylaspartate (NAA) was measured in the human normal-appearing corpus callosum on a 7 tesla MRI scanner, comparing 15 MS patients and 14 healthy controls. We found that NAA parallel diffusivity is lower in MS $(p=0.030)$ and inversely correlated with both water parallel diffusivity $(p=0.020)$ and clinical severity $(p=0.015)$. Interpreted in the context of previous experiments, our findings provide preliminary evidence that DTS can distinguish axonopathy from other processes such as inflammation, edema, demyelination, and gliosis. By detecting reduced diffusion of NAA parallel to axons in white matter, DTS may thus be capable of distinguishing axonal disruption in MS in the setting of increased parallel diffusion of water, which is commonly observed in MS but pathologically nonspecific.

\section{Introduction}

Multiple sclerosis (MS) is an immune-mediated demyelinating disease of the CNS. It has become accepted that neuroaxonal pathology is responsible for much of the progressive disability associated with chronic MS (Trapp et al., 1998; Tallantyre et al., 2010). The long-term effects of neurodegeneration can be detected in the form of tissue loss (atrophy), whereas ongoing neurodegeneration, manifested as neurons that are damaged but still intact, is exceedingly difficult to measure in vivo.

$\mathrm{N}$-acetylaspartate (NAA) is detectable in proton magnetic resonance spectroscopy (MRS) due to its exceptionally high concentration in the CNS. It has been hailed as a specific neuroaxonal marker because brain NAA is almost exclusively localized within neurons (Simmons et al., 1991). The metabolic role of NAA is believed to be twofold (Moffett et al., 2007). NAA is synthe-

Received Dec. 27, 2011; revised Feb. 1, 2012; accepted March 21, 2012.

Author contributions: E.T.W., I.R., P.B.B., P.C., D.H., and D.S.R. designed research; E.T.W., I.R., and D.S.R. performed research; E.T.W., I.R., A.T., and C.K.J. contributed unpublished reagents/analytic tools; E.T.W., I.R., and D.S.R. analyzed data; E.T.W. and D.S.R. wrote the paper.

This study was supported by the Intramural Research Program of the National Institute of Neurological Disorders and Stroke, as well as by grants from NIH (P41RR015241), Bayer Schering Pharma (to P.C. and D.H.), and the Johns Hopkins Institute for Clinical and Translational Research to support data collection. We thank Terri Brawner, Kathie Kahl, and Ivana Kusevic for their attention to detail and exceptional subject care; and Peter van Zijl, Joe Gillen, and Richard Edden for technical support.

Correspondence should be addressed to Dr. DanielS. Reich, Translational Neuroradiology Unit, NIH/NINDS, Building 10, Room 5C103, 10 Center Drive MSC 1400, Bethesda, MD 20892-1400. E-mail: daniel.reich@nih.gov.

DOI:10.1523/JNEUROSCI.0044-12.2012

Copyright $\odot 2012$ the authors $\quad 0270-6474 / 12 / 326665-05 \$ 15.00 / 0$ sized in neurons, where it facilitates mitochondrial metabolism by preventing product inhibition of the tricarboxylic acid cycle. Additionally, NAA is exported to oligodendrocytes for rapid breakdown into precursors of fatty acid and steroid synthesis that are critical for myelin formation. Consequently, decreased NAA concentration in neurodegenerative diseases has been interpreted both as neuronal loss and neuronal dysfunction.

Diffusion tensor spectroscopy (DTS) (van Zijl et al., 1994; Kroenke et al., 2004; Ellegood et al., 2006; Upadhyay et al., 2007, 2008; Kan et al., 2011) combines features of both diffusion tensor imaging (DTI) and MRS, allowing measurement of the diffusion properties of intracellular metabolites. DTI is a sensitive technique that provides information about microscopic structural features of anisotropic tissues such as white matter (WM) tracts. Unfortunately, the pathologic specificity of DTI is limited because the measured signal is derived from water protons, which are found in all tissue compartments (including inflammatory cells, myelin, neurons, and glia). In contrast, MRS is neurochemically and compartmentally specific but provides no microstructural information. However, localized MRS can deliver tissue-specific structural information when combined with diffusion sensitization for compartment-specific metabolites such as NAA. DTS measurements of NAA thus provide specific information about axonal microstructure and might consequently serve as a useful marker of axonal integrity in the complex mixture of pathologies that characterizes MS. 
Here, we compare the diffusion properties of NAA and water in the corpus callosum (CC) between MS patients and healthy controls (HC) at 7 tesla (T). CC damage is frequent and tightly linked with clinical disability in MS (Ozturk et al., 2010). From a neuroanatomic point of view, the CC is a good structure in which to apply DTS because it is relatively homogeneous, contains fiber bundles that are predominantly oriented in the same direction, and is large enough to harbor a spectroscopy voxel. Acquisition at $7 \mathrm{~T}$ makes it possible to more rapidly achieve adequate signal-to-noise ratio.

\section{Materials and Methods}

The Johns Hopkins Medicine Institutional Review Board approved this study. Fifteen MS patients ( 7 female, 8 male) from the Johns Hopkins MS Clinic and 14 HC (6 female, 8 male) from the university community provided informed consent. Patients had not experienced clinical relapse within 3 months of enrollment. All patients and seven HC underwent clinical testing, including measures of motor function (25-Foot Walk, 9-Hole Peg test) and cognitive function [Paced Auditory Serial Addition Test (PASAT)], and patients were assigned Expanded Disability Status Scale (EDSS) scores.

MRI and MRS data were obtained with a 7T Philips Achieva scanner with $40 \mathrm{mT} / \mathrm{m}$ maximum gradients (Philips Medical Systems), quadrature volume transmit, and 16- or 32channel receive head coils (Nova Medical). $\mathrm{T}_{1}{ }^{-}$ weighted images were acquired for spectroscopy volume of interest (sVOI) positioning and tissue segmentation: 3D MPRAGE sequence with TR, $4.1 \mathrm{~ms}$; TE, $1.85 \mathrm{~ms}$; flip angle, $7.0^{\circ}$; resolution, $1 \mathrm{~mm}$ isotropic; scan time, $92 \mathrm{~s}$; SENSE factor, 2 (anterior-posterior, AP) $\times 3$ (right-left, $\mathrm{RL}) . \mathrm{T}_{1}$-weighted volumes were processed in MIPAV (McAuliffe et al., 2001), including inhomogeneity correction (Sled et al., 1998), skullstripping with SPECTRE (Carass et al., 2011), and segmentation into WM, gray matter (GM), and CSF using the FANTASM (Pham and Prince, 1999). A mask of the sVOI was applied to the segmented volume to make a tissue (no CSF) mask (tVOI). CC cross-sectional area (CCarea) was measured in MIPAV by drawing regions of interest on the three midsagittal $\mathrm{T}_{1}$-weighted slices and averaging the area of these regions. $\mathrm{T}_{2}$-weighted FLAIR images were acquired and qualitatively examined for lesion detection: 3D MP-FLAIR sequence (Visser et al., 2010), TR, 8000 $\mathrm{ms}$; TI, $2175 \mathrm{~ms}$; TE, $292 \mathrm{~ms}$; resolution, $1 \mathrm{~mm}$ isotropic; scan time, 8 min; SENSE factor, $2 \times 3$.

Diffusion-weighted spectra were acquired from a $3.6 \mathrm{~cm}^{3}$ sVOI $(3.0$ $\mathrm{cm} \mathrm{AP} \times 1.5 \mathrm{~cm} \mathrm{RL} \times 0.8 \mathrm{~cm}$ foot-head) positioned and angled on $\mathrm{T}_{1}$-weighted images to include portions of the genu and anterior body of the normal-appearing CC and to minimize partial volume effects of CSF and GM (Fig. 1a,c). NAA diffusion measurements were obtained by incorporating bipolar diffusion gradients into a point-resolved spectroscopic sequence (Upadhyay et al., 2007; Kan et al., 2011): TR, 2000 ms; TE, 120 ms; bandwidth, $3 \mathrm{kHz} ; 2048$ points; 32-40 averages; scan time, $15 \mathrm{~min}$. Frequency-selective excitation/dephasing water suppression was optimized to allow reliable NAA quantification while retaining enough residual water signal for zero-order phase correction before spectral averaging. A non-water-suppressed spectrum, with the transmitter frequency on water, was acquired from the sVOI for eddy current correction. Diffusion weighting was applied in six non-coplanar directions using two $b$ values in addition to $b=0: 440 \mathrm{~s} / \mathrm{mm}^{2}$ and either 2250
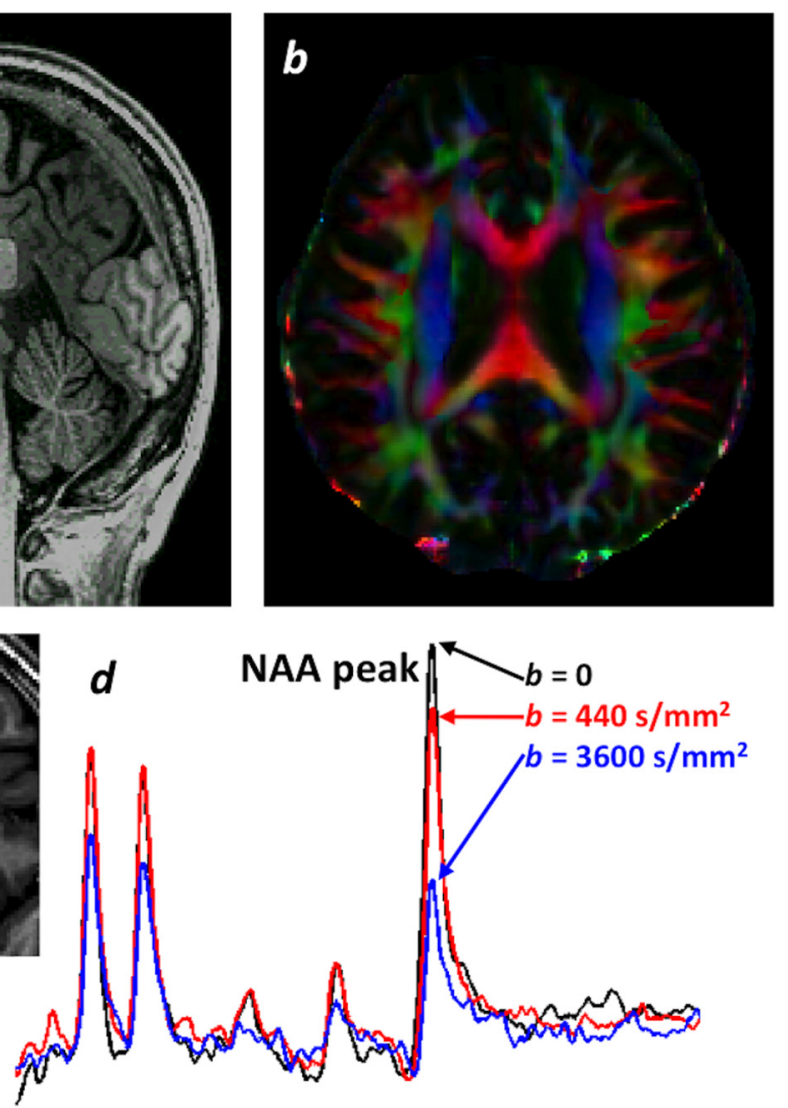

ra. a, c, Sagittal (a) and coronal (c) T1-weighted images demonstrating voxel location (red box). $\boldsymbol{b}$, DTI axial color map. $\boldsymbol{d}$, Nondiffusion-weighted spectrum (black) and diffusion-weighted spectra with gradient direction primarily parallel to fiber tract (red and blue).

$\mathrm{s} / \mathrm{mm}^{2}$ (11 scans) or $3600 \mathrm{~s} / \mathrm{mm}^{2}$ (22 scans). Individual and averaged spectra were phase- and frequency-drift corrected in MATLAB (Mathworks). The averaged spectrum for each diffusion direction and $b$ value combination was quantified with LCModel (Provencher, 1993). Due to low signal-to-noise ratio (SNR) at the highest $b$ values, NAA and $\mathrm{N}$-acetylaspartylglutamate (NAAG) were not well separated, so the NAA + NAAG values (Cramér-Rao lower bounds $<15 \%$ ) were used for diffusion analysis. The diffusion tensor was estimated and diagonalized to yield fractional anisotropy (FA), mean diffusivity (MD), parallel diffusivity ( $\lambda_{\|}=\lambda_{1}$; diffusion parallel to the primary fiber orientation) and perpendicular diffusivity $\left(\lambda_{\perp}=\left(\lambda_{2}+\lambda_{3}\right) / 2\right.$; diffusion orthogonal to the primary fiber orientation). $\mathrm{tNAA} / \mathrm{tCr}$ was estimated from the ratio of NAA + NAAG to creatine + phosphocreatine from the $b=0$ spectrum.

A DTI volume was acquired for comparison with DTS: Spin Echo Segmented Echo Planar Imaging sequence; TR, 12,643 ms; TE, $82 \mathrm{~ms} ; b$ value, $800 \mathrm{~s} / \mathrm{mm}^{2}$; resolution, $2.0 \mathrm{~mm}$ isotropic; 30 directions; scan time, $7 \mathrm{~min}$; SENSE factor, 3 (AP). In MIPAV, the individual diffusionweighted volumes were rigidly registered to the $b=0$ image, which was registered (affine) to the $\mathrm{T}_{1}$-weighted volume. The diffusion-weighted data were then analyzed in two ways: at native resolution with averaging within the tVOI (avgDTI) and at sVOI resolution (svDTI). For svDTI, the tVOI mask was applied to each diffusion-weighted volume to extract an average diffusion-weighted value for each direction before tensor fitting. The svDTI analysis controls for the macrostructural effects of CC curvature on diffusion values and is used instead of DTS-derived water spectra, which suffered from partial volume averaging with CSF.

All statistical analyses were done with STATA (StataCorp). $p$ values $<0.05$ were considered statistically significant. Group differences in age and sex were assessed with $t$ tests. Linear regression was used to characterize associations between age, MRS, DTI, DTS, continuous clinical measures, and disease status. For this, a mixed-effects model was used to account for the random effects of repeated scans for four HC. When 
Table 1. Demographics and MR measures

\begin{tabular}{|c|c|c|c|}
\hline & Patients $(n=15)$ & Controls $(n=14)$ & $p$ value \\
\hline Age in years (range) & $44(28-55)$ & $38(24-56)$ & $n s^{*}$ \\
\hline Sex (number of women) & 7 & 6 & $n s^{*}$ \\
\hline \multicolumn{4}{|l|}{ MS type } \\
\hline Relapsing remitting & 12 & $\mathrm{n} / \mathrm{a}$ & \\
\hline Secondary progressive & 1 & $\mathrm{n} / \mathrm{a}$ & \\
\hline Primary progressive & 2 & $\mathrm{n} / \mathrm{a}$ & \\
\hline Median EDSS score (range) & $3(1-6.5)$ & $\mathrm{n} / \mathrm{a}$ & \\
\hline Disease duration in years (range) & $8(1-26)$ & $\mathrm{n} / \mathrm{a}$ & \\
\hline CC cross-sectional area $\left(\mathrm{mm}^{2}\right)$ & 575 & 654 & 0.017 \\
\hline GM proportion of tV0I & 0.28 & 0.26 & ns \\
\hline tNAA/tCr (NAA + NAAG:Cr + PCr ratio) & 1.47 & 1.84 & $<0.001$ \\
\hline \multicolumn{4}{|l|}{ Fractional anisotropy } \\
\hline NAA & 0.53 & 0.58 & ns \\
\hline Water & 0.43 & 0.49 & 0.016 \\
\hline \multicolumn{4}{|l|}{ Mean diffusivity $\left(\mu \mathrm{m}^{2} / \mathrm{ms}\right)$} \\
\hline NAA & 0.15 & 0.18 & ns \\
\hline Water & 1.48 & 1.22 & $<0.001$ \\
\hline \multicolumn{4}{|l|}{ Parallel diffusivity $\left(\lambda_{\|}\right)\left(\mu \mathrm{m}^{2} / \mathrm{ms}\right)$} \\
\hline NAA & 0.25 & 0.31 & 0.030 \\
\hline Water & 2.13 & 1.86 & $<0.00$ \\
\hline \multicolumn{4}{|l|}{ Perpendicular diffusivity $\left(\lambda_{\perp}\right)\left(\mu \mathrm{m}^{2} / \mathrm{ms}\right)$} \\
\hline NAA & 0.11 & 0.11 & ns \\
\hline Water & 1.15 & 0.90 & $<0.001$ \\
\hline
\end{tabular}

Mean values and regression $p$ values unless otherwise noted. Water values from avgDTI analysis. ns, Not significant; $\mathrm{n} / \mathrm{a}$, not applicable. *Student's $t$ test.

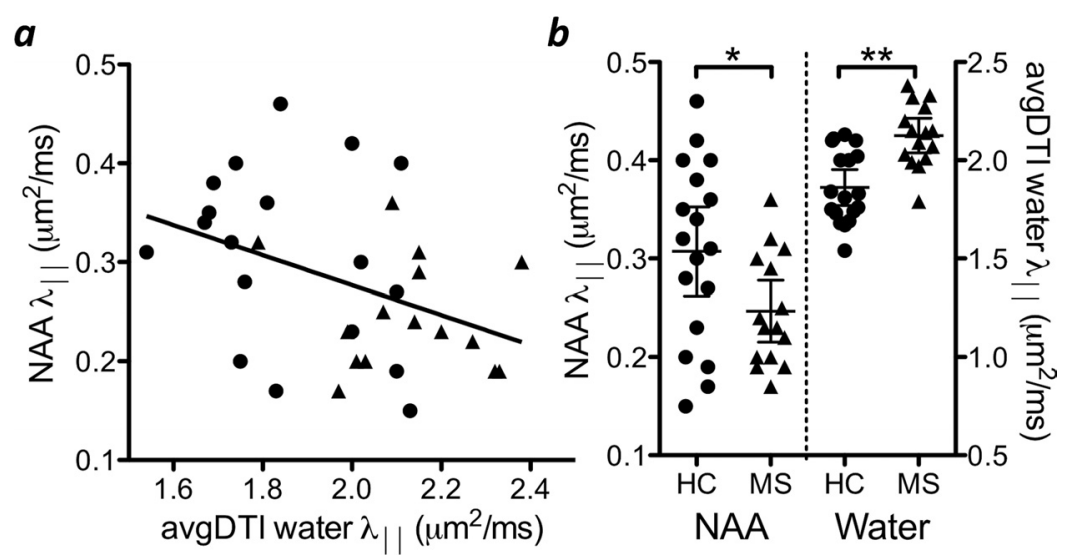

Figure 2. Parallel diffusivity $\left(\lambda_{\|}\right)$of NAA and water. $\boldsymbol{a}, \mathrm{NAA} \lambda_{\|}$is negatively correlated with avgDTI water $\lambda_{\|}$(all subjects, $R^{2}=$ $0.16, p=0.02)$. $\boldsymbol{b}$, NAA $\lambda_{\|}$and avgDTl water $\lambda_{\|}$for MS patients and HC. ${ }^{*} p<0.05,{ }^{* *} p<0.001$; error bars represent $95 \%$ confidence intervals.

applicable, the regression coefficient (RC), coefficient of determination $\left(R^{2}\right)$, and $p$ value are reported. Correlations $(\rho)$ of measures with EDSS were assessed with the Spearman rank correlation test.

\section{Results}

The HC group was not significantly different from the MS group for age and sex. Twelve patients were on immunomodulatory treatment; none had received steroids within $30 \mathrm{~d}$ of scanning. Patients demonstrated smaller CCarea and lower tNAA/tCr ( Table 1) in accordance with previous studies (Davie et al., 1994; Riccitelli et al., 2011). tNAA/tCr was negatively correlated with age for MS ( $\mathrm{RC}=-0.012 /$ year, $p=0.007)$ and $\mathrm{HC}(\mathrm{RC}=$ -0.011 year, $p=0.026$ ), as seen previously (Haga et al., 2009). There was no significant difference between groups for use of a higher $b$ value of 2250 versus $3600 \mathrm{~mm} / \mathrm{s}^{2}$ or between NAA diffusion values measured with different higher $b$ values.
NAA diffusion data are summarized in Table 1. NAA $\lambda_{\|}$was the only DTS measure significantly different between groups (Fig. 2b). Note that no macroscopic lesions were identified in the sVOI on visual inspection of the $T_{1}$-weighted or FLAIR images. Diffusion-weighted spectroscopy has been studied previously in healthy human volunteers at lower field strengths (Posse et al., 1993; Kroenke et al., 2004; Ellegood et al., 2006; Upadhyay et al., 2007) and 7T (Kan et al., 2011), yielding comparable diffusivity findings.

All water diffusion results are derived from the DTI acquisition. svDTI values were correlated with, but lower than, their avgDTI counterparts- e.g., avgDTI FA versus svDTI FA ( RC = $\left.0.70, p<0.001, R^{2}=0.56\right)$, avgDTI MD versus svDTI MD $(\mathrm{RC}=$ $\left.0.77, p<0.001, R^{2}=0.83\right)$ - and svDTI FA (mean $\left.=0.28\right)$ was lower than avgDTI FA (mean $=0.47)$ (paired $t$ test, $p<0.0001$ ). This difference was expected due to macroscopic curvature of the $\mathrm{CC}$ within the tVOI, as combining diffusion data across a volume with a variety of axonal orientations will decrease FA. The mean avgDTI FA is low compared with previous studies (Reich et al., 2010), probably due to partial volume averaging with GM in the tVOI.

NAA MD and $\lambda_{\|}$, as well as avgDTI FA, were positively correlated with $\mathrm{tNAA} / \mathrm{tCr}(p<0.001, p=0.013$, and $p=0.032$, respectively). In contrast, avgDTI MD, $\lambda_{\|}$, and $\lambda_{\perp}$ were negatively correlated with $\mathrm{tNAA} / \mathrm{tCr}($ all $p<0.001)$. There was also a significant negative correlation between NAA $\lambda_{\|}$and water $\lambda_{\|}(p=0.022$; Fig. $2 a)$. A logistic regression of disease status on NAA $\lambda_{\|}$and water $\lambda_{\|}$was not significant for either regressor.

Finally, several NAA measures, but no measures of water diffusion, were related to clinical measures of disease severity. Lower tNAA/tCr was associated with longer times to complete the 9-hole peg test $(p<0.001)$ and lower PASAT scores $(p=$ $0.042)$. Lower NAA MD $(\rho=-0.59, p=$ $0.020)$, NAA $\lambda_{\|}(\rho=-0.61, p=0.015$; Fig. 3), and NAA $\lambda_{\perp}(\rho=-0.53, p=$ 0.045 ) values were related to higher EDSS scores (more clinical disability).

\section{Discussion}

These results demonstrate that DTS is clinically relevant in MS and brings specificity to MRS and DTI measures of neurodegeneration. In MS, neurodegeneration occurs in the setting of multiple pathological processes in different tissue compartments. Because NAA is primarily found within axons in WM, NAA diffusion properties should be a more specific marker of WM integrity than water diffusion alone. Consistent with previous studies, and discussed further below, we found higher water $\lambda_{\|}$and $\lambda_{\perp}$ in MS compared with HC, indicating nonspecific microstructural changes in these patients with low disease burden. By contrast, we found that NAA $\lambda_{\|}$was lower in MS and inversely correlated with water $\lambda_{\|}$. Additionally, only DTS measures were correlated with clinical disability as measured by EDSS. These results lend support to diffusion measurements of NAA being a more specific marker for previous and ongoing axonopathy in MS than water diffusion alone.

The intra-axonal location of NAA, together with findings that intact cell membranes are relatively impermeable to NAA and neuronal export is controlled by a transporter (Moffett et al., 


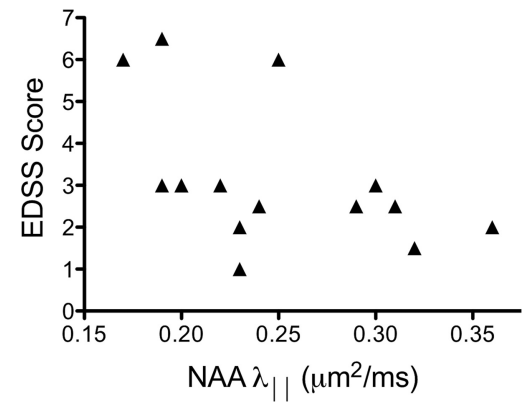

Figure 3. NAA parallel diffusivity $\left(\lambda_{\|}\right)$is correlated with clinical severity of disease ( $\rho=$ $-0.61, p=0.015)$.

2007), leads to the prediction that, under normal physiological circumstances, NAA should preferentially diffuse parallel to the long axis of axons with a similar anisotropy to intra-axonal water. It has been shown at $3 \mathrm{~T}$ that NAA FA is more sensitive than water FA to microstructural organization of axons across two segments of the CC (Upadhyay et al., 2007). This result offers support for the notion that NAA diffusion is a more specific probe of intraaxonal physiology than water diffusion and can therefore add additional information.

There is mounting evidence that axonal loss and neurodegeneration are responsible for progressive and irreversible neurological disability in MS (Gonen et al., 2000; Trapp and Nave, 2008). However, there are no specific therapies or in vivo measures for neurodegeneration in MS. Axonal degeneration in MS is known to occur in lesions and normal-appearing WM (NAWM) (Trapp et al., 1998; Kutzelnigg et al., 2005). Histological characterizations of diffuse WM injury in MS provide a picture of axonal degeneration similar to animal models and other human pathologies, e.g., axonal swellings and end bulbs, $\beta$-amyloid precursor protein accumulation, and neurofilament alterations (Mahad et al., 2009). Measurements of [NAA] in CSF are similar between MS and HC, suggesting that neurons are not leakier for NAA in MS (Teunissen et al., 2009). Based on simulated diffusion models of axon damage in complex geometries (Budde and Frank, 2010; Landman et al., 2010), we expect that the structural disruptions described in axonal degeneration would restrict the diffusion of NAA along the length of axons and would be associated with lower NAA $\lambda_{\|}$, as seen in our data. Decreased NAA $\lambda_{\|}$ may confirm the presence of axonopathy in the face of overall increased water diffusion due to inflammation, edema, and demyelination in MS. This is highlighted by the correlation between clinical status and NAA $\lambda_{\|}$(but not water $\lambda_{\|}$), since disability may be more closely related to neuroaxonal pathology than to inflammation.

DTI has been heavily studied in MS patients and animal models with conflicting results. Most MS studies have demonstrated increased water MD, $\lambda_{\|}$, and $\lambda_{\perp}$ and decreased water FA in both lesions and NAWM, suggesting nonspecific microstructural damage (Werring et al., 1999; Bammer et al., 2000; Reich et al., 2010). In contrast, studies in mouse models of inflammation, such as allergic/autoimmune encephalomyelitis (DeBoy et al., 2007; Budde et al., 2009), and demyelination, such as cuprizone (Xie et al., 2010), have demonstrated decreased water $\lambda_{\|}$in conjunction with axon injury and degeneration.

A potentially important difference between DTI studies in MS and animal models is the timing of imaging in relation to clinical symptoms or inciting events. Animal studies with serial imaging consistently show early decreased water $\lambda_{\|}$, corresponding to histological evidence of axonopathy, followed by normalized or increased water $\lambda_{\|}$. This pattern of DTI changes was borne out in a human optic neuritis study, which benefitted from a known, discrete clinical onset. In patients with optic neuritis, low baseline water $\lambda_{\|}$was correlated with poor visual acuity and a thinned retinal nerve fiber layer at 6 months (Naismith et al., 2012). However, this study represents the exception rather than the rule: as axonopathy is ongoing and almost always found in the setting of inflammation and edema in MS, DTI is, in most cases, unable to reliably identify the presence of axonopathy.

Cross-sectional MRS studies in MS, including this one, have demonstrated decreased [NAA] compared with HC in lesions, NAWM, and the whole brain, suggesting axonal loss (Fu et al., 1998; Gonen et al., 2000; Oh et al., 2004). Decreased [NAA] has been found in NAWM and GM in the earliest stages of the disease and in those with relatively little disability (Filippi et al., 2003; Inglese et al., 2004; Wattjes et al., 2007; Rigotti et al., 2011). However, longitudinal studies in NAWM have not been able to detect declining [NAA] in patients with MS over the course of 2-3 years (Sajja et al., 2008). Interestingly, MRS MS studies have seen [NAA] drop dramatically within acute lesions only to rise to nearnormal levels in follow-up scans, suggesting that some decreases in [NAA] can be attributed to reversible metabolic insults (Davie et al., 1994) or perhaps to dilutional effects related to edema. To distinguish [NAA] decreases due to neuron or axon loss from metabolic issues, some studies have used regression modeling to adjust [NAA] for DTI measures of axon integrity (Cader et al., 2007; Ciccarelli et al., 2010). While these studies support a role for mitochondrial impairment in MS, it remains difficult to judge by MRS measures of [NAA] whether axons are damaged or destroyed.

DTS measurements are limited by low concentrations of metabolites compared with water, lowering the SNR of DTS relative to DTI. The SNR and measurement variability of DTS could have been improved by acquiring and averaging more spectra, but acquisition time was limited because this pilot project was part of a larger study. Although high magnetic field and strong gradients allowed us to use a relatively small voxel containing primarily coherent WM fibers, our measurements still have partial-volume effects of GM and vasculature. However, when DTS is applied to a specific fiber tract with a known orientation, most of the variability in the DTS measures can be attributed to microstructural properties of the WM tract. The high correlation between svDTI and avgDTI measures suggests that partial-volume and macroscopic curvature effects may not be important limiting factors. Finally, the overlap of NAA $\lambda_{\|}$between MS and HC (Fig. 2b) indicates that DTS measurements in NAWM are unlikely to be highly sensitive for detecting the presence of diseased tissue in individual cases. Therefore, it is important to interpret the finding of low NAA $\lambda_{\|}$in one particular brain region in the context of other MRI measures across the brain.

In conclusion, DTS may be a relatively specific marker of neurodegeneration in MS, in contrast to more conventionally acquired MRI measures such as $\mathrm{T}_{1}$ gadolinium contrast enhancement (indicating inflammatory blood-brain barrier opening), focal $\mathrm{T}_{2}$ hyperintensity (a range of pathologies including inflammation, edema, demyelination, gliosis, and leukomalacia), and magnetization transfer (changes in myelination and water content). In MS, DTS changes may help interpret findings from more sensitive techniques, such as DTI, elucidating their pathophysiological significance. Future studies will further explore the specificity of NAA diffusion by in-depth analysis of the natural history of DTS measures in MS in animal models of demyelination and neurodegeneration, where histopathological correlates can be examined, and in autopsy tissue. 


\section{References}

Bammer R, Augustin M, Strasser-Fuchs S, Seifert T, Kapeller P, Stollberger R, Ebner F, Hartung HP, Fazekas F (2000) Magnetic resonance diffusion tensor imaging for characterizing diffuse and focal white matter abnormalities in multiple sclerosis. Magn Reson Med 44:583-591.

Budde MD, Frank JA (2010) Neurite beading is sufficient to decrease the apparent diffusion coefficient after ischemic stroke. Proc Natl Acad Sci U S A 107:14472-14477.

Budde MD, Xie M, Cross AH, Song SK (2009) Axial diffusivity is the primary correlate of axonal injury in the experimental autoimmune encephalomyelitis spinal cord: a quantitative pixelwise analysis. J Neurosci 29:2805-2813.

Cader S, Johansen-Berg H, Wylezinska M, Palace J, Behrens TE, Smith S, Matthews PM (2007) Discordant white matter $N$-acetylasparate and diffusion MRI measures suggest that chronic metabolic dysfunction contributes to axonal pathology in multiple sclerosis. Neuroimage 36:19-27.

Carass A, Cuzzocreo J, Wheeler MB, Bazin PL, Resnick SM, Prince JL (2011) Simple paradigm for extra-cerebral tissue removal: algorithm and analysis. Neuroimage 56:1982-1992.

Ciccarelli O, Toosy AT, De Stefano N, Wheeler-Kingshott CA, Miller DH, Thompson AJ (2010) Assessing neuronal metabolism in vivo by modeling imaging measures. J Neurosci 30:15030-15033.

Davie CA, Hawkins CP, Barker GJ, Brennan A, Tofts PS, Miller DH, McDonald WI (1994) Serial proton magnetic resonance spectroscopy in acute multiple sclerosis lesions. Brain 117:49-58.

DeBoy CA, Zhang J, Dike S, Shats I, Jones M, Reich DS, Mori S, Nguyen T, Rothstein B, Miller RH, Griffin JT, Kerr DA, Calabresi PA (2007) High resolution diffusion tensor imaging of axonal damage in focal inflammatory and demyelinating lesions in rat spinal cord. Brain 130:2199-2210.

Ellegood J, Hanstock CC, Beaulieu C (2006) Diffusion tensor spectroscopy (DTS) of human brain. Magn Reson Med 55:1-8.

Filippi M, Bozzali M, Rovaris M, Gonen O, Kesavadas C, Ghezzi A, Martinelli V, Grossman RI, Scotti G, Comi G, Falini A (2003) Evidence for widespread axonal damage at the earliest clinical stage of multiple sclerosis. Brain 126:433-437.

Fu L, Matthews PM, De Stefano N, Worsley KJ, Narayanan S, Francis GS, Antel JP, Wolfson C, Arnold DL (1998) Imaging axonal damage of normal-appearing white matter in multiple sclerosis. Brain 121:103-113.

Gonen O, Catalaa I, Babb JS, Ge Y, Mannon LJ, Kolson DL, Grossman RI (2000) Total brain $N$-acetylaspartate: a new measure of disease load in MS. Neurology 54:15-19.

Haga KK, Khor YP, Farrall A, Wardlaw JM (2009) A systematic review of brain metabolite changes, measured with $1 \mathrm{H}$ magnetic resonance spectroscopy, in healthy aging. Neurobiol Aging 30:353-363.

Inglese M, Ge Y, Filippi M, Falini A, Grossman RI, Gonen O (2004) Indirect evidence for early widespread gray matter involvement in relapsingremitting multiple sclerosis. Neuroimage 21:1825-1829.

Kan HE, Techawiboonwong A, van Osch MJ, Versluis MJ, Deelchand DK, Henry PG, Marjańska M, van Buchem MA, Webb AG, Ronen I (2011) Differences in apparent diffusion coefficients of brain metabolites between grey and white matter in the human brain measured at 7 T. Magn Reson Med 67:1203-1209.

Kroenke CD, Ackerman JJ, Yablonskiy DA (2004) On the nature of the NAA diffusion attenuated MR signal in the central nervous system. Magn Reson Med 52:1052-1059.

Kutzelnigg A, Lucchinetti CF, Stadelmann C, Brück W, Rauschka H, Bergmann M, Schmidbauer M, Parisi JE, Lassmann H (2005) Cortical demyelination and diffuse white matter injury in multiple sclerosis. Brain 128:2705-2712.

Landman BA, Farrell JA, Smith SA, Reich DS, Calabresi PA, van Zijl PC (2010) Complex geometric models of diffusion and relaxation in healthy and damaged white matter. NMR Biomed 23:152-162.

Mahad DJ, Ziabreva I, Campbell G, Lax N, White K, Hanson PS, Lassmann H, Turnbull DM (2009) Mitochondrial changes within axons in multiple sclerosis. Brain 132:1161-1174.

McAuliffe MJ, Lalonde FM, McGarry D, Gandler W, Csaky K, Trus BL (2001) Medical image processing, analysis and visualization in clinical research. In: 14th IEEE Symposium on Computer-Based Medical Systems, pp 381-386.

Moffett JR, Ross B, Arun P, Madhavarao CN, Namboodiri AM (2007) $\mathrm{N}$-acetylaspartate in the CNS: from neurodiagnostics to neurobiology. Prog Neurobiol 81:89-131.
Naismith RT, Xu J, Tutlam NT, Lancia S, Trinkaus K, Song SK, Cross AH (2012) Diffusion tensor imaging in acute optic neuropathies: predictor of clinical outcomes. Arch Neurol 69:65-71.

Oh J, Pelletier D, Nelson SJ (2004) Corpus callosum axonal injury in multiple sclerosis measured by proton magnetic resonance spectroscopic imaging. Arch Neurol 61:1081-1086.

Ozturk A, Smith SA, Gordon-Lipkin EM, Harrison DM, Shiee N, Pham DL, Caffo BS, Calabresi PA, Reich DS (2010) MRI of the corpus callosum in multiple sclerosis: association with disability. Mult Scler 16:166-177.

Pham DL, Prince JL (1999) Adaptive fuzzy segmentation of magnetic resonance images. IEEE Trans Med Imaging 18:737-752.

Posse S, Cuenod CA, Le Bihan D (1993) Human brain: proton diffusion MR spectroscopy. Radiology 188:719-725.

Provencher SW (1993) Estimation of metabolite concentrations from localized in vivo proton NMR spectra. Magn Reson Med 30:672-679.

Reich DS, Ozturk A, Calabresi PA, Mori S (2010) Automated vs conventional tractography in multiple sclerosis: variability and correlation with disability. Neuroimage 49:3047-3056.

Riccitelli G, Rocca MA, Forn C, Colombo B, Comi G, Filippi M (2011) Voxelwise assessment of the regional distribution of damage in the brains of patients with multiple sclerosis and fatigue. Am J Neuroradiol 32:874-879.

Rigotti DJ, Gonen O, Grossman RI, Babb JS, Falini A, Benedetti B, Filippi M (2011) Global N-acetylaspartate declines even in benign multiple sclerosis. Am J Neuroradiol 32:204-209.

Sajja BR, Narayana PA, Wolinsky JS, Ahn CW, PROMiSe Trial MRSI Group (2008) Longitudinal magnetic resonance spectroscopic imaging of primary progressive multiple sclerosis patients treated with glatiramer acetate: multicenter study. Mult Scler 14:73-80.

Simmons ML, Frondoza CG, Coyle JT (1991) Immunocytochemical localization of $\mathrm{N}$-acetyl-aspartate with monoclonal antibodies. Neuroscience 45:37-45.

Sled JG, Zijdenbos AP, Evans AC (1998) A nonparametric method for automatic correction of intensity nonuniformity in MRI data. IEEE Trans Med Imaging 17:87-97.

Tallantyre EC, Bø L, Al-Rawashdeh O, Owens T, Polman CH, Lowe JS, Evangelou N (2010) Clinico-pathological evidence that axonal loss underlies disability in progressive multiple sclerosis. Mult Scler 16:406-411.

Teunissen CE, Iacobaeus E, Khademi M, Brundin L, Norgren N, KoelSimmelink MJA, Schepens M, Bouwman F, Twaalfhoven HA, Blom HJ, Jakobs C, Dijkstra CD (2009) Combination of CSF N-acetylaspartate and neurofilaments in multiple sclerosis. Neurology 72:1322-1329.

Trapp BD, Nave KA (2008) Multiple sclerosis: an immune or neurodegenerative disorder? Annu Rev Neurosci 31:247-269.

Trapp BD, Peterson J, Ransohoff RM, Rudick R, Mörk S, Böø L (1998) Axonal transection in the lesions of multiple sclerosis. N Engl J Med 338:278-285.

Upadhyay J, Hallock K, Erb K, Kim DS, Ronen I (2007) Diffusion properties of NAA in human corpus callosum as studied with diffusion tensor spectroscopy. Magn Reson Med 58:1045-1053.

Upadhyay J, Hallock K, Ducros M, Kim DS, Ronen I (2008) Diffusion tensor spectroscopy and imaging of the arcuate fasciculus. Neuroimage 39:1-9.

van Zijl PCM, Davis D, Moonen CTW (1994) Diffusion spectroscopy in living systems. In: NMR in physiology and biomedicine (Gillies RJ, ed), pp 185-198. San Diego: Academic.

Visser F, Zwanenburg JJ, Hoogduin JM, Luijten PR (2010) High-resolution magnetization-prepared 3D-FLAIR imaging at 7.0 Tesla. Magn Reson Med 64:194-202.

Wattjes MP, Harzheim M, Lutterbey GG, Klotz L, Schild HH, Träber F (2007) Axonal damage but no increased glial cell activity in the normalappearing white matter of patients with clinically isolated syndromes suggestive of multiple sclerosis using high-field magnetic resonance spectroscopy. Am J Neuroradiol 28:1517-1522.

Werring DJ, Clark CA, Barker GJ, Thompson AJ, Miller DH (1999) Diffusion tensor imaging of lesions and normal-appearing white matter in multiple sclerosis. Neurology 52:1626-1632.

Xie M, Tobin JE, Budde MD, Chen CI, Trinkaus K, Cross AH, McDaniel DP, Song SK, Armstrong RC (2010) Rostrocaudal analysis of corpus callosum demyelination and axon damage across disease stages refines diffusion tensor imaging correlations with pathological features. J Neuropathol Exp Neurol 69:704-716. 